\title{
CD8 Naive T Cell Counts Decrease Progressively in HIV-infected Adults
}

\author{
Mario Roederer, J. Gregson Dubs, Michael T. Anderson, Paul A. Raju, Leonore A. Herzenberg, \\ and Leonard A. Herzenberg \\ Department of Genetics, Stanford University School of Medicine, Stanford, California 94305
}

\begin{abstract}
We show here that $\mathrm{CD8}$ naive $\mathrm{T}$ cells are depleted during the asymptomatic stage of HIV infection. Although overall CD8 $T$ cell numbers are increased during this stage, the naive CD8 $T$ cells are progressively lost and fall in parallel with overall CD4 $\mathrm{T}$ cell counts. In addition, we show that naive CD4 $\mathrm{T}$ cells are preferentially lost as total CD4 cell counts fall. These findings, presented here for adults, and in the accompanying study for children, represent the first demonstration that HIV disease involves the loss of both CD4 $T$ cells and CD8 $T$ cells. Furthermore, they provide a new insight into the mechanisms underlying the immunodeficiency of $\mathrm{HIV}$-infected individuals, since naive $\mathbf{T}$ cells are required for all new $\mathbf{T}$ cell-mediated immune responses.

Studies presented here also show that the well-known increase in total CD8 counts in most HIV-infected individuals is primarily due to an expansion of memory cells. Thus, memory CD8 $T$ cells comprise over $80 \%$ of the $T$ cells in PBMC from individuals with $<200 \mathrm{CD} 4 / \mu \mathrm{l}$, whereas they comprise roughly $15 \%$ in uninfected individuals. Since the naive and memory subsets have very different functional activities, this altered naive/memory $T$ cell representation has significant consequences for the interpretation of data from in vitro functional studies. (J. Clin. Invest. 1995. 95:2061-2066.) Key words: memory T cells - CD45RA CD62L • absolute $\mathrm{T}$ cell counts $\bullet$ immunodeficiency
\end{abstract}

\section{Introduction}

Recent flow cytometric studies have identified several phenotypically and functionally distinct subsets of CD4 and CD8 T cells (1-5). Functional studies have assigned specific roles to some of these subsets. In particular, the terms memory and naive distinguish subsets which either contain or do not contain (respectively) long-lived cells capable of mounting an immediate response to a specific antigen. In vitro studies have also demonstrated that these subsets have distinct functional capacities: in general, the memory subsets do not proliferate as well as naive subsets in response to generic mitogenic stimuli; however, the memory subsets produce a wider variety and greater amounts of many cytokines $(1,6,7)$.

Address correspondence to Leonard A. Herzenberg, Department of Genetics, Stanford University, Beckman B007, Stanford, CA 94305. Phone: 415-723-5054; FAX: 415-725-8564.

Received for publication 16 June 1994 and in revised form 14 November 1994.

J. Clin. Invest.

(C) The American Society for Clinical Investigation, Inc.

0021-9738/95/05/2061/06 \$2.00

Volume 95, May 1995, 2061-2066
Studies characterizing changes in $\mathrm{T}$ cell representation in AIDS have generally focused on the decrease in total CD4 counts that occurs as the disease progresses. Indeed, for over a decade, the specific loss of these cells has been the most commonly used surrogate marker for disease progression. Unlike CD4 $\mathrm{T}$ cell counts, absolute CD8 counts have been shown to rise early after HIV infection and maintain a relatively steady level until very late in the disease (8) when CD4 counts drop below $100 / \mu 1(9)$.

Studies here, which focus on the functionally distinct subsets within both $\mathrm{CD} 4$ and $\mathrm{CD} 8 \mathrm{~T}$ cell lineages, challenge this paradigm. We demonstrate a profound loss of naive $\mathrm{T}$ cells, both CD4 and CD8, in HIV-infected adults. Similarly, in studies presented in an accompanying manuscript (10), we demonstrate that CD8 naive T cells are also lost in HIV-infected children. In addition, we show that the early increase in total CD8 counts is due primarily to the expansion of the memory CD8 subsets.

Previous attempts to quantitate the relative levels of naive and memory subsets showed little or no preferential loss of either subset during the progression of $\operatorname{AIDS}(8,11-15)$; however, the combination of reagents used in these previous studies is insufficient to resolve the naive $T$ cells from other subsets. In contrast, the specific three-color immunofluorescence FACS ${ }^{\circledR}$ methodology we use here, i.e., the combination of CD45RA and CD62L together with CD4 or CD8, uniquely identifies and enumerates the naive and memory $T$ cell subsets. Thus, we were able to determine the representation of these subsets in over $250 \mathrm{HIV}$-infected individuals and thereby demonstrate the selective loss of naive $\mathrm{T}$ cells during the progression of HIV disease.

\section{Methods}

Human samples. We recruited 266 HIV-infected adults from the San Francisco area. Since this study was part of a clinical trial in which an entry criterion was $<500 \mathrm{CD} 4 \mathrm{~T}$ cells per microliter, our cohort is weighted towards infected individuals with fewer CD4 T cells than the general infected population. Also excluded from participation were patients who had concurrent opportunistic infections or were taking very large amounts of antioxidants, vitamins, or minerals. The infection status of each individual was confirmed by anti-p24 ELISA. In addition, we recruited 44 HIV-uninfected adults, in good health, as control subjects. All clinical trial subjects signed an informed consent form.

From each HIV-infected patient, blood was drawn by venipuncture for FACS ${ }^{\circledR}$ analysis; for 242 of the 266 patients, blood was drawn for a complete blood count and an absolute CD4 and CD8 count (performed by an accredited commercial laboratory). From control subjects, only blood for FACS ${ }^{\circledR}$ analysis was drawn. All samples were prepared within $8 \mathrm{~h}$ of the draw.

Reagents. All fluorochrome-conjugated monoclonals were obtained from PharMingen (San Diego, CA). Ficol-Paque was obtained from Pharmacia AB (Uppsala, Sweden). Biotin, flavin-deficient RPMI-1640 (hereafter, RPMI) was obtained from Irvine Scientific (Santa Ana, CA). Other chemicals were obtained from Sigma Chemical Co. (St. Louis, MO).

FACS ${ }^{\circledR}$ analysis. PBMC were prepared from $6 \mathrm{ml}$ of heparinized 

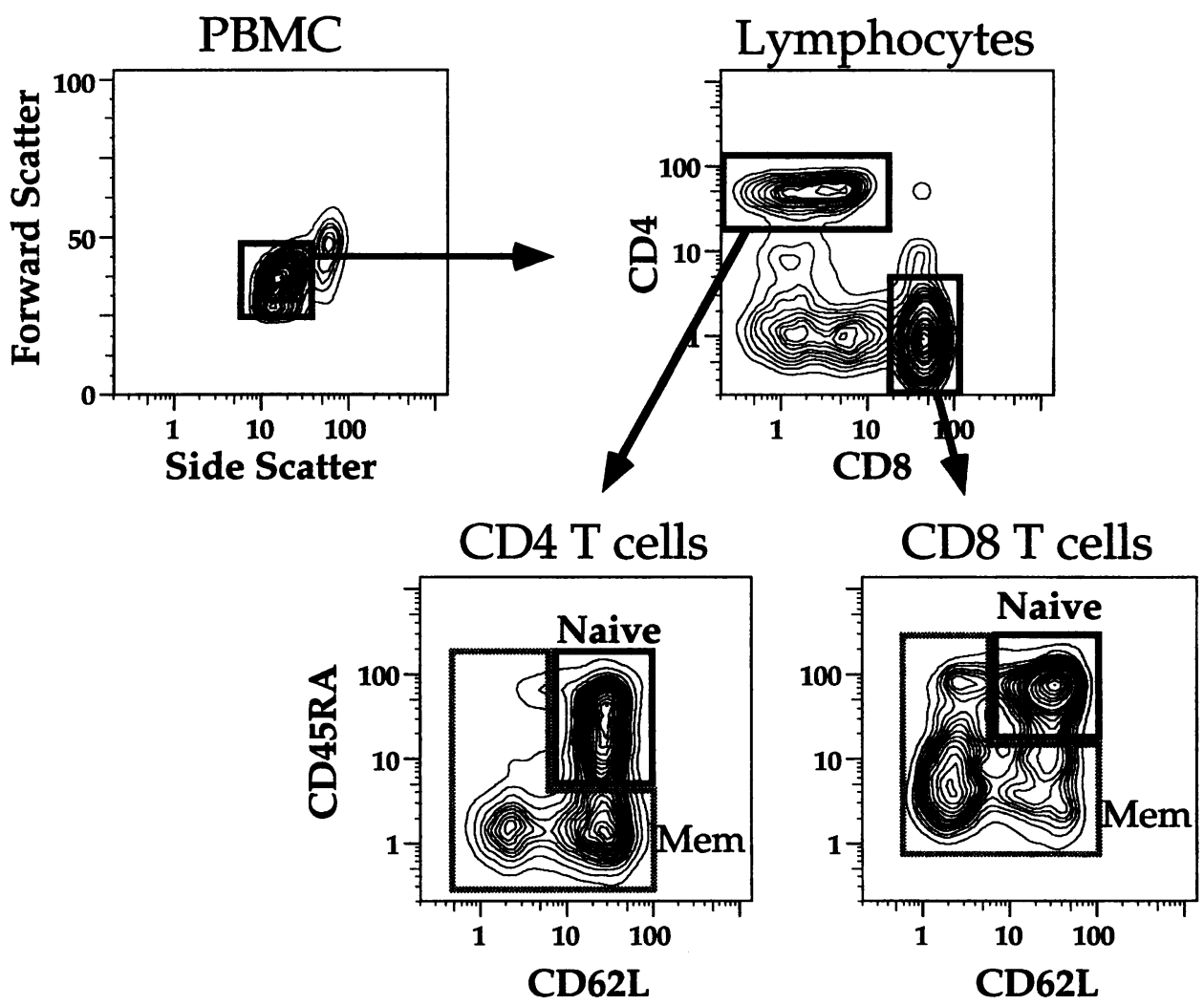

Figure 1. T cell subset phenotypes. Progressively gated PBMC sample from a healthy adult. The lymphocytes are selected by scatter gates (top left). Within lymphocytes, CD4 and CD8 $\mathrm{T}$ cells can be discriminated (top right). Also evident are NK cells (dim CD8 staining) and B cells (negative for both CD4 and CD8). Within CD4 or CD8 cells (boxes) are several subsets (bottom panels). The naive subset (dark box) of both CD4 and CD8 lineages are defined as $\mathrm{CD} 4 \mathrm{RA}^{+}$and $\mathrm{CD} 62 \mathrm{~L}^{+}$; the remaining cells ( gray box) comprise the memory subsets. Within CD8 T cells (bottom right) are at least three memory subsets: cells expressing only one of CD45RA or CD62L, and those expressing neither. Within CD4 $\mathrm{T}$ cells (bottom left) are two major memory subsets: both are CD45RA ${ }^{-}$, but one is $\mathrm{CD}^{2} 2 \mathrm{~L}^{+}$and the other is $\mathrm{CD}^{2} 2 \mathrm{~L}^{-}$. In healthy adults, there are very few CD4 T cells with the CD45RA ${ }^{+}$ $\mathrm{CD}_{62} \mathrm{~L}^{-}$phenotype (averaging $5 \%$ of total CD4); however, in HIV-infected adults, this population becomes much more prevalent (averaging $15 \%$ of all $\mathrm{CD} 4$ and as much as $40-50 \%$ in some patients; see Fig. $2 B$ for an example). blood by Ficol-Paque density centrifugation. For analyses in this report, we used the following antibody combinations: $(a)$ fluorescein-conjugated (FITC) CD62L, phycoerythrin (PE) ${ }^{1}$ CD45RA, and CyChrome (Cy5-PE) CD8; ( b ) FITC CD62L, PE CD45RA, and Cy5-PE CD4; (c) FITC CD11a, PE CD45RA, and Cy5-PE CD4; $(d)$ FITC CD11a, PE CD45RA, and Cy5-PE CD8; $(e)$ FITC CD3, PE CD8, and Cy5-PE CD4; and $(f)$ FITC CD14, PE CD16, and Cy5-PE CD45. Concentrated antibody preparations were titered in combinations with unconjugated antibody to obtain saturating reagents that were on-scale in fluorescence. Enough reagent had been premixed to study the entire cohort that we report on here, ensuring comparable fluorescence distributions across all experiments.

After staining, cells were washed three times in biotin, flavin-deficient RPMI-1640 and then resuspended in 0.5\% paraformaldehyde in RPMI. Flow cytometric analysis was performed on a dual laser (argon $360 \mathrm{~nm}$, argon $488 \mathrm{~nm}$ ) FACStar Plus ${ }^{\circledR}$ (Becton Dickinson Immunocytometry Systems, San Jose, CA) interfaced to a VAX 6300 computer (Digital Computer, Maynard, MA). For each cell, data for forward scatter, side scatter, and the fluorescences of fluorescein $(515-545-\mathrm{nm}$ bandpass filter), phycoerythrin (570-600 nm), and Cy5-PE (650-690 $\mathrm{nm}$ ) were collected. For each stain, data from 50,000 cells were collected and analyzed by FACS-Desk ${ }^{\circledR}$ software (16). For subset frequencies, a lymphocyte gate was used. In addition, gates uniquely identifying CD4 or CD8 T cells and their subsets (as shown in Fig. 1) were applied. Absolute numbers of subsets of CD4 or CD8 T cells were found by multiplying their representation by the absolute subset counts obtained at the clinical laboratory. For instance, the number of naive CD4 per microliter of whole blood is calculated by multiplying the fraction of CD4 $\mathrm{T}$ cells which are naive (from FACS ${ }^{\circledR}$ gating) by the absolute CD4 count per microliter of blood. We did not obtain CD4 and CD8

1. Abbreviations used in this paper: CTL, cytotoxic T lymphocyte; PE, phycoerythrin. counts for the control (HIV-uninfected) population. Values in Table I were derived from the same clinical laboratory that performed the absolute counts for HIV-infected adults in our study.

Statistical analyses. For analysis and display of statistical comparisons, we used JMP for the Apple Macintosh (SAS Institute, Cary, NC). Comparisons of distributions were performed by the nonparametric twosample Wilcoxon rank test.

\section{Results}

Both CD4 and CD8 $\mathrm{T}$ cells from blood can be further subdivided into subsets based on surface phenotypes. The combination of CD62L (L-selectin) and CD45RA defines at least four phenotypically distinct subsets in each lineage (Fig. 1). Functional studies have shown that naive $T$ cells belong to the subsets which stain brightly with both of these antibodies, and dimly for CD11a (LFA-1 $\alpha)(1,4,5)$. Three other subsets of cells, consisting of the other combinations of CD62L and CD45RA expression, comprise the memory compartment $(4,5)$.

Naive CD4 $\mathrm{T}$ cells differ from naive CD8 $\mathrm{T}$ cells in that the CD45RA expression on CD8 $\mathrm{T}$ cells is uniformly higher than on CD4 T cells. Indeed, even the CD45RA negative CD8 cells still display low levels of CD45RA. Since some of these cells express as much CD45RA as CD4 naive cells, the frequencies of naive cells of each lineage must be determined independently (i.e., using CD3 to identify $\mathrm{T}$ cells, it is not possible to use a gate based on CD45RA and CD62L expression that will include all CD8 and CD4 naive T cells and will not include memory $\mathrm{T}$ cells). Thus, enumerating $\mathrm{CD} 8$ and $\mathrm{CD} 4$ naive $\mathrm{T}$ cells in PBMC samples requires simultaneous FACS ${ }^{\circledR}$ measurement of three cell surface antigens in two separate tubes (Fig. 1), i.e., CD8 (or CD4), together with CD45RA and CD62L. 


\begin{tabular}{|c|c|c|c|c|}
\hline \multirow[b]{2}{*}{ Cells } & \multicolumn{3}{|c|}{$\mathrm{HIV}^{+}(\mathrm{CD} 4 / \mu \mathrm{l})^{\ddagger}$} & \multirow[b]{2}{*}{$\mathrm{HIV}^{-\ddagger}$} \\
\hline & $0-200$ & $200-500$ & $>500$ & \\
\hline \multicolumn{5}{|c|}{ Fraction of $\mathrm{T}$ cells that are naive $(\%)$} \\
\hline CD8 & $12(7-18)$ & $16(11-24)$ & $23(16-33)$ & $52(41-59)$ \\
\hline CD4 & $27(18-37)$ & $39(30-49)$ & $45(30-57)$ & $50(41-58)$ \\
\hline \multicolumn{5}{|c|}{ Absolute number of cells $/ \mu 1$ of blood } \\
\hline Total CD8 & $520(310-780)$ & $790(580-1110)$ & $750(590-1100)$ & $620(330-910)^{\S}$ \\
\hline Naive CD8 & $58(34-94)$ & $120(93-160)$ & $175(140-230)$ & $320^{\| \prime}$ \\
\hline Naive CD4 & $18(7-37)$ & $120(85-178)$ & $250(160-230)$ & $490^{\prime}$ \\
\hline
\end{tabular}

* Numbers presented are the median values for each group (either percent of each lineage that is naive in the upper portion of the table, or cells/ $\mu \mathrm{l}$ for each subset in the lower portion). The interquartile range is given in parentheses. ${ }^{\ddagger}$ Number of individuals in each group: $\mathrm{HIV}^{-}=44 ; \mathrm{HIV}^{+}$, $0-200=109 ; 200-500=105 ;>500=28$. ${ }^{8}$ This is the value for all $\mathrm{HIV}^{-}$adults seen at the same clinic which performed our absolute counts, including subjects not in our cohort. "This value is based on the total CD8 count (620) multiplied by the average percent of CD8 T cells which are naive in uninfected adults from our study (52\%). 'The average value for total CD4 count (980), for all $\mathrm{HIV}^{-}$adults seen at the clinic, multiplied by the fraction of CD4 $\mathrm{T}$ cells which are naive $(50 \%)$.

FACS ${ }^{\circledR}$ plots in Fig. 2 show the relative frequencies of $\mathrm{T}$ cell subsets in PBMC from representative HIV-infected and uninfected adults. In agreement with results from our pediatric study (10), these plots show that HIV-infected adults lose a significant fraction of naive $\mathrm{T}$ cells of both CD4 and CD8 lineages. This loss is visible as a decrease in the representation of the CD45RA ${ }^{+} \mathrm{CD} 2 \mathrm{~L}^{+}$cells. Absolute counts (cells per microliter of blood) of these subsets, calculated by combining
A. CD8

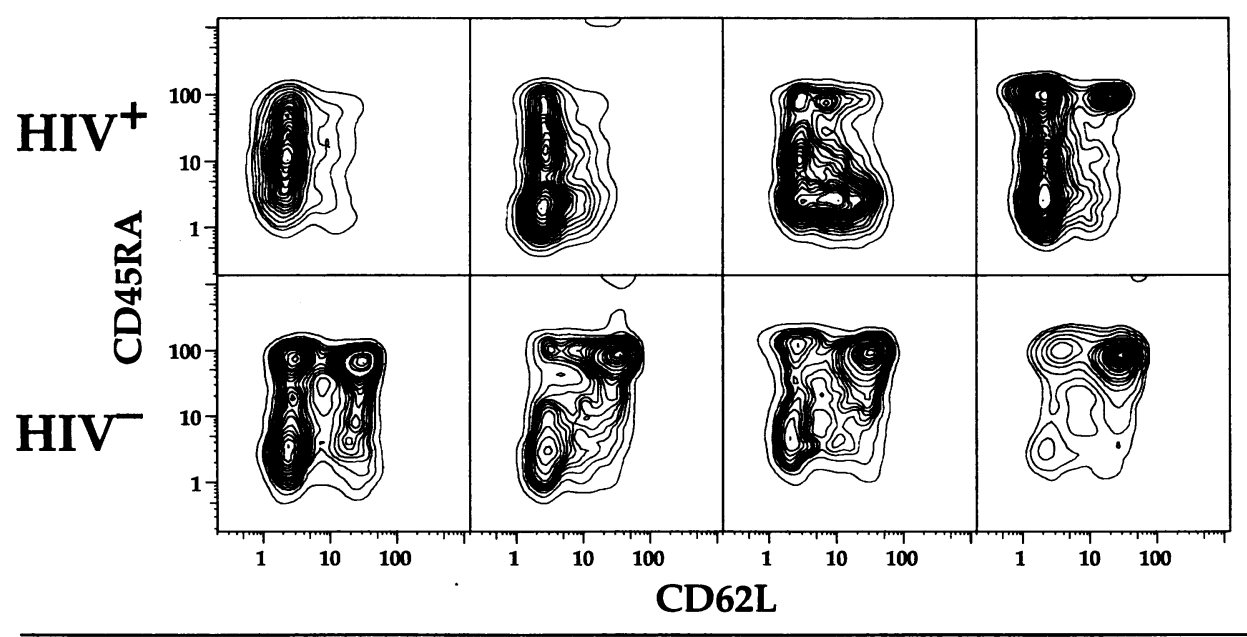

B. CD4

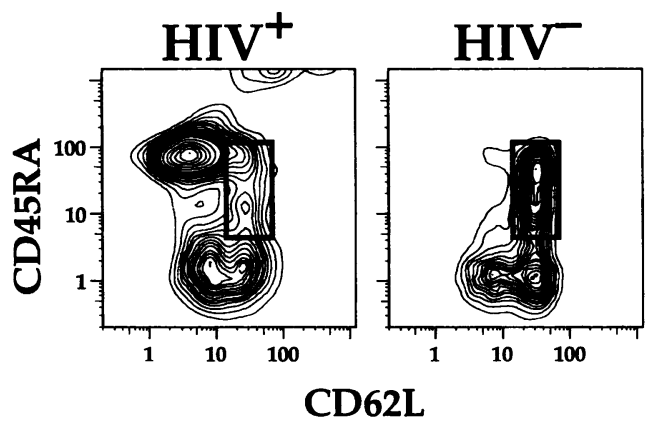

Figure 2. Examples of $\mathrm{T}$ cell subsets in healthy and HIV-infected adults.

(A) CD8 T cells. Four examples each from the HIV-negative cohort (bottom) and the HIV-positive cohort (top) were selected to show the range of naive $T$ cell representation in these groups, and are arranged in order of increasing naive CD8 $\mathrm{T}$ cell representation (left to right). Only CD8 T cells are shown (based on gating similar to that shown in Fig. 1). The loss of the naive subset in the infected individuals is seen by the decrease of the CD45RA ${ }^{+} \mathrm{CD}_{2} \mathrm{~L}^{+}$subset, and a corresponding increase in memory. Note that an increase of memory cells in one $\mathrm{HIV}^{+}$adult may reflect expansion of a different subset than in another $\mathrm{HIV}^{+}$adult (e.g., compare the third and fourth top panels). While there is a marked variation in the representation of the various subsets within individuals (both HIV-infected and uninfected), the phenotypes of these subsets are constant. (B) CD4 $T$ cells. A comparison of an uninfected individual (right) and an HIVinfected individual (left) is shown, with the box denoting the position of the naive cells. (The exact phenotype of naive CD4 T cells is invariant in all uninfected adults and in those infected adults who still retain some naive cells.) This HIV-infected adult has a large proportion of CD45RA ${ }^{+}$CD4 $\mathrm{T}$ cells. However, these are not naive $\mathrm{T}$ cells, since they have at least several characteristics distinguishing them as memory: as shown in the figure, they are considerably brighter for CD45RA than naive CD4 cells and they are low for CD62L expression. Furthermore, they express HLA-DR and CD38 activation markers and contain very low intracellular glutathione (data not shown). 

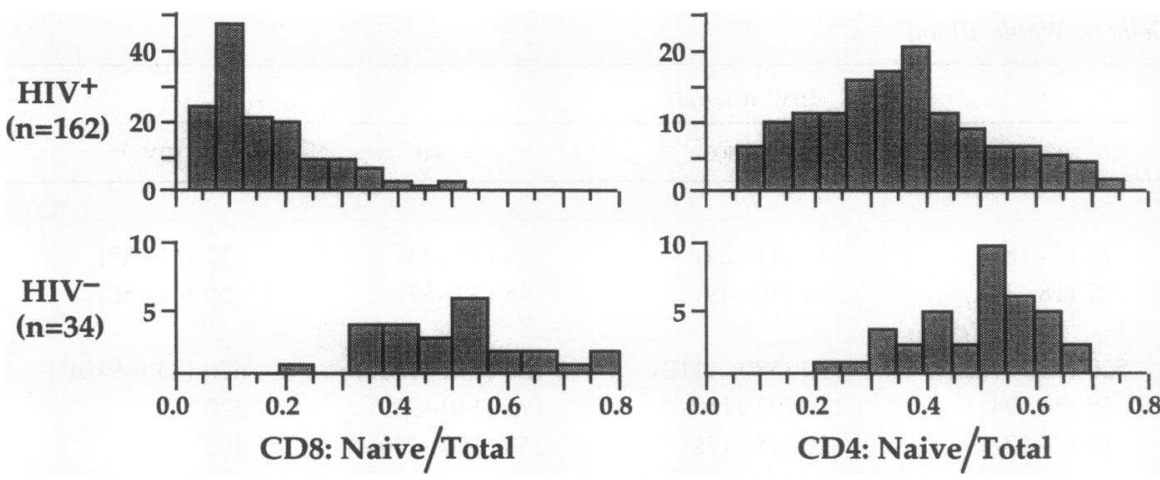

Figure 3. Representation of naive $\mathrm{T}$ cells within the CD4 and CD8 lineages. In both lineages, there is a substantial decrease in the representation of naive $\mathrm{T}$ cells and a corresponding increase in memory. This is much more dramatic in CD8 $\mathrm{T}$ cells (left) than CD4 T cells (right): $90 \%$ of HIV-infected adults (top) have a lower fraction of naive CD8s than almost all uninfected adults (bottom). The decrease in the CD4 naive T cells may be even greater than these data show, because of the overlap in expression of CD45RA on memory CD4 $\mathrm{T}$ cells and naive CD4 $\mathrm{T}$ cells (e.g., see Fig. $2 B$ ). This overlap results in an overestimate of naive CD4 representation for infected adults. Finally, these data were calculated from the frequencies of CD62L ${ }^{+}, \mathrm{CD}^{2} \mathrm{R} \mathrm{A}^{+}$ $T$ cells; however, virtually identical results were obtained for each subject by determining the frequency of the CD11a-dim, $\mathrm{CD}^{2} 5 \mathrm{RA}^{+}$cells of both CD4 and CD8 T subsets.

these FACS ${ }^{\circledR}$ frequency data with complete blood count data, show a similar decline (see below).

Previous researchers have found little consistent change in memory and naive representation among $T$ cells between infected and uninfected adults $(8,11-15)$. However, these studies used only CD62L or only CD45RA to identify subsets. Thus, the contribution of a memory subset to this single phenotype confounded quantitation of naive cells. This is borne out in the examples shown in Fig. 2. All of the HIV-infected individuals shown have very few naive cells. Even so, the third individual has a considerable fraction of memory $\mathrm{CD}^{2} 2 \mathrm{~L}^{+}$cells and the fourth has a considerable fraction of memory CD45RA ${ }^{+}$cells. Measurement of only one or the other marker would therefore not have revealed the naive cell loss in these individuals.

The decreased frequency of naive $\mathrm{T}$ cells is particularly dramatic in the CD8 lineage (Fig. 3). 50\% of CD8 T cells in healthy adults typically belong to the naive subset; however, in most HIV-infected adults, $<15 \%$ of CD8 cells are naive. This change in naive/memory representation does not necessarily reflect a decrease in the absolute numbers of the naive cells. In fact, among the HIV-infected individuals with the highest CD4 counts, absolute naive CD8 counts are sometimes in the normal range, but significant elevation of the memory count drives the ratio of naive/memory well below the normal ratio of one half (Table I and Fig. 4).

In general, as total CD4 counts fall, naive CD8 T cell counts also fall (Fig. $4 \mathrm{~A}$ ). Since the absolute number of CD4 $\mathrm{T}$ cells per microliter of blood provides a reasonable indication (surrogate marker) for the progression of AIDS, we interpret this data as indicating that the naive CD8 $\mathrm{T}$ cells are selectively lost as HIV disease progresses. While there is a loose correlation between these two counts, it is also apparent than many individuals have either considerably more, and others fewer, naive CD8 T cells than would be predicted by their total CD4 count. Therefore, the naive CD8 $\mathrm{T}$ cell count may provide a useful marker to distinguish individuals who are very different immunologically and yet would be stratified together by the use of CD4 counts.

Naive CD4 T cells are also preferentially lost during HIV disease progression (Fig. $4 \mathrm{C}$ ). While more than half of CD4 $\mathrm{T}$ cells in healthy adults are in the naive subset, about onefourth are naive in HIV-infected adults with CD4 counts under $200 / \mu$ l (Table I). Since the preferential loss of naive CD4 T cells parallels the loss of naive CD8 T cells, a similar mechanism might account for the decrease in both naive subsets.

\section{Discussion}

We have used three-color immunophenotyping to address the impact of HIV infection on the representation of defined $\mathrm{T}$ cell subsets in adults (here) and children (10). By specifically distinguishing the naive $\mathrm{T}$ cells from those in various memory subsets, we found a preferential loss of the naive $\mathrm{T}$ cells that correlates with progression of HIV disease (as measured by the decline of total CD4 T cells). Significantly, the loss of the naive cells begins relatively early after infection, when these individuals are otherwise asymptomatic and still have substantial numbers of CD4 $\mathrm{T}$ cells.

These findings reveal a previously unsuspected complexity in the dynamics of $\mathrm{T}$ cell representation in HIV-infected individuals. Despite the progressive decrease of naive CD8 T cells, the overall CD8 $\mathrm{T}$ cell number persists at elevated levels until very late in the disease. The increased numbers of CD8 T cells must therefore reflect a selective increase in memory CD8 T cells. Since naive $\mathrm{T}$ cells are memory precursors, their loss, which precedes the eventual loss of memory cells, may contribute substantially to the eventual loss of the total CD8 population.

The early disappearance of the naive CD8 cells is particularly surprising, since most discussions of $\mathrm{T}$ cell loss in HIV infection have centered around the specific infectability of CD4bearing cells. However, since Bonyhadi and colleagues (17) have shown that the thymocytes expressing both CD8 and CD4 are infectable by HIV and will die when infected, the loss of naive CD8 T cells could well be due to the depletion of these double positive precursors. Alternatively, loss of thymopoietic capability could be due to a general destruction of the thymus or a progressive loss of the bone marrow's capacity to produce thymocyte progenitors. Hypotheses based on general failure of thymopoiesis are attractive since they could also explain the preferential loss of naive CD4 T cells (Fig. 4).

In any case, the loss of the naive $T$ cells has important consequences for the development of immune responses in HIV-infected individuals. As the naive subsets disappear, there will be a progressive inability to mount responses to novel antigens, which may well result in high susceptibility to opportunistic infections. Furthermore, this loss will compromise the 
A

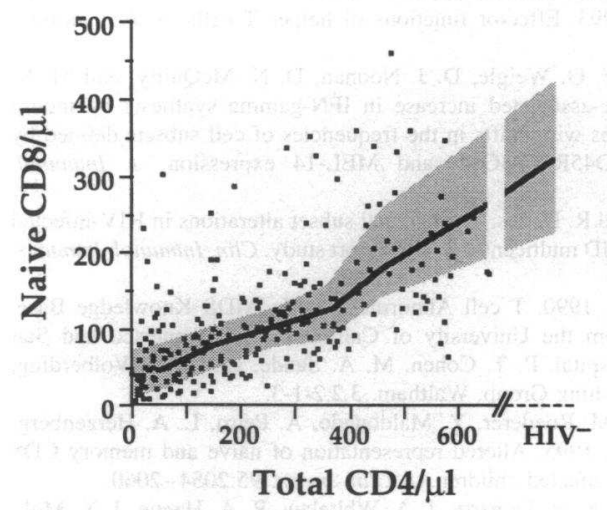

B

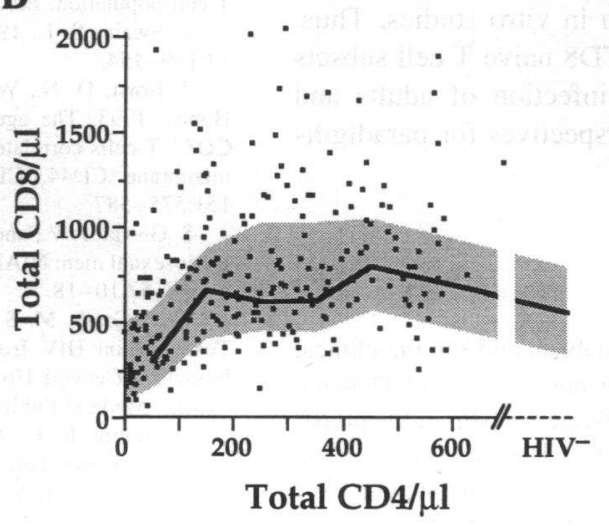

C

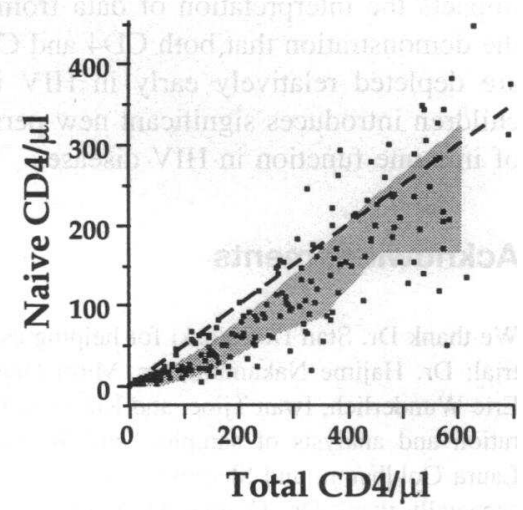

Figure 4. Correlation of naive $\mathrm{T}$ cell representation with absolute CD4 count: naive cells in both CD8 and CD4 lineages are lost preferentially during the progression of HIV disease. $(A)$ The absolute number of naive CD8 T cells is plotted against the absolute total CD4 count. Each dot represents one individual. The line connects the median values for individuals grouped by CD4 counts (each group is based on a range of $100 \mathrm{CD} 4$ cells $/ \mu 1$ ). The shaded area is the interquartile range for the groups. The range for uninfected adults is from Table I. (B) The total CD8 counts are plotted as in A. Most infected adults have more total CD8 cells than uninfected adults. Since the number of naive $\mathrm{T}$ cells is declining, the increase is due solely to the expansion of memory CD8 T cells. $(C)$ The absolute number of naive CD4 T cells is plotted. The shaded area is the interquartile range; the line connecting medians is not drawn. If naive and memory CD4 T cells were lost from HIV-infected individuals at the same rate, then the shaded region would be centered on the dashed line. (The slope of the dashed line, 0.5 , is the fraction of CD4 T cells which are naive in uninfected adults; see Table I.) Since the distribution actually curves well below this line, naive CD4 T cells are lost preferentially to memory CD4 $\mathrm{T}$ cells in HIV-infected individuals.

ability to deal with the constantly mutating virus itself: novel strains, which are immunogenically unique, will encounter less resistance from $\mathrm{T}$ cell immunity than early in the progression of disease.

The impairment of immune function due to the loss of naive T cells from HIV-infected adults and children also has important consequences for therapeutic strategies for AIDS. Since responses to novel antigens have to arise (by definition) from the naive compartment, the magnitude or effectiveness of such responses is necessarily dependent on the availability of naive $\mathrm{T}$ cells. Therefore, an HIV-infected individual with no naive $\mathrm{T}$ cells is likely to fail to respond to any primary immunization involving $\mathrm{T}$ cells, be it therapeutic vaccination or otherwise. As a practical matter, this means that therapeutic vaccination trials can be severely compromised by failure to control for the variability in the number of naive $\mathrm{T}$ cells in study subjects.

Similar considerations apply to therapies involving immunomodulators and ex vivo expansion of cytotoxic $\mathrm{T}$ lymphocytes (CTL) for therapeutic reinfusion. Since the representation of CTL precursors and CTL effector cells changes dramatically during the progression of AIDS, the effectiveness of these therapies (or the ability to expand cells capable of carrying out immunity) may vary significantly between patients, due to the variable representation of CD8 subsets. Thus, our findings suggest that therapeutic trials for vaccines and immunomodulating methodologies should be stratified with respect to naive $\mathrm{T}$ cell count in addition to total CD4 count. Efficacy trials for therapeutic drugs should similarly benefit from this type of stratification, which will eliminate the confounding variable of nonresponsiveness due to the lack of naive $T$ cells.

Finally, the loss of naive $T$ cells coupled with the relative overrepresentation of memory $\mathrm{CD} 8 \mathrm{~T}$ cells introduces the necessity for reexamination of all conclusions drawn from functional studies of PBMC from HIV-infected individuals. Memory subsets are the primary producers of certain cytokines such as IL-
4, IL-10, and $\gamma$-IFN; naive subsets tend to produce mainly IL-2. Therefore, simply changing the representation of these functionally distinct subsets will change the cytokine profile of a culture.

For example, cultures of PBMC from a typical HIV-infected individual with $<100 \mathrm{CD} 4 / \mu \mathrm{l}$ will have very few naive $\mathrm{T}$ cells, and an overrepresentation of memory CD8 cells. Thus, even if the functions of naive and memory cells are equivalent in HIVinfected and uninfected individuals, PBMC cultures from the HIV-infected individuals will produce less IL-2 and more IL-4 than in comparable cultures from the uninfected individuals. Similarly, since naive $\mathrm{T}$ cells proliferate better in response to mitogen than do memory cells, the PBMC cultures from HIVinfected individuals with few naive $T$ cells will proliferate less in response to, e.g., phytohemagglutinin. Unfortunately, this means that previous demonstrations of these types of functional differences between PBMC from individuals at different stages of disease (or between PBMC from infected versus uninfected donors ) could merely reflect differences in the underlying representation of naive and memory cells.

The loss of T cells and the impairment of $\mathrm{T}$ cell functionality are hallmarks of HIV disease. However, such deficiencies are not necessarily restricted to this disease. For example, chronic alcohol consumption frequently results in a loss of $\mathrm{T}$ cell function and a decreased representation of naive $\mathrm{T}$ cell subsets (18). Thus, the mechanisms underlying the naive $\mathrm{T}$ cell loss in HIV disease may be unique to HIV disease or reflect general immunoregulatory processes that HIV infection sets in motion.

In summary, regardless of how naive $T$ cells are lost or the specificity of the naive $\mathrm{T}$ cell loss to HIV disease, this loss has important consequences for the disease itself and the ways in which we study it. At a functional level, the progressive loss of naive cells contributes to the overall immunodeficiency in the disease by decreasing the ability to respond to novel antigens. At a methodological level, the selective loss of the naive subset, 
and the corresponding overrepresentation of memory subsets, impacts the interpretation of data from in vitro studies. Thus, the demonstration that both CD4 and CD8 naive T cell subsets are depleted relatively early in HIV infection of adults and children introduces significant new perspectives for paradigms of immune function in HIV disease.

\section{Acknowledgments}

We thank Dr. Stan Deresinski for helping establish and run the clinical trial; Dr. Hajime Nakamura, Dr. Mitra Dependra, Dr. Steve DeRosa, Eric Wunderlich, Iwan Tjioe, and Kai-Uwe Belge for help in the preparation and analysis of samples; and William Clawson, David Hook, Laura Goldbaum, and Sharon O'Leary for expert nursing support. We especially thank Dr. Thomas Merigan for making the Stanford Center for AIDS Research available for these studies, and Dr. David Parks and the other members of the Stanford Shared FACS ${ }^{\oplus}$ facility staff for flow cytometry support.

Mario Roederer is a Senior Fellow of the Leukemia Society of America. This work was supported in part by National Institutes of Health grants LM-04836, AI-31770, and CA-42509 together with a special supplement to CA-42509 for the clinical trial.

\section{References}

1. Sanders, M. E., M. W. Makgoba, and S. Shaw. 1988. Human naive and memory T cells. Immunol. Today. 9:195-199.

2. Sohen, S., D. Rothstein, T. Tallman, D. Gaudette, S. Schlossman, and C. Morimoto. 1990. The functional heterogeneity of $\mathrm{CD}^{+}$cells defined by antiCD45RA (2H4) and anti-CD29 (4B4) antibodies. Cell. Immunol. 128:314-328.

3. De Jong, R., M. Brouwer, F. Miedema, and R. A. van Lier. 1991. Human $\mathrm{CD}^{+} \mathrm{T}$ lymphocytes can be divided into $\mathrm{CD}^{+} 5 \mathrm{RA}^{+}$and $\mathrm{CD} 45 \mathrm{RO}^{+}$cells with different requirements for activation and differentiation. J. Immunol. 146:20882094.

4. Picker, L. J., J. R. Treer, D. B. Ferguson, P. A. Collins, D. Buck, and L. W. Terstappen. 1993. Control of lymphocyte recirculation in man. I. Differential regulation of the peripheral lymph node homing receptor $\mathrm{L}$-selectin on $\mathrm{T}$ cells during the virgin to memory cell transition. J. Immunol. 150:1105-1121.

5. Okumura, M., Y. Fujii, Y. Takeuchi, K. Inada, K. Nakahara, and H. Ma- tsuda. 1993. Age-related accumulation of LFA- $1^{\text {high }}$ cells in a CD8 ${ }^{+}$CD45RA ${ }^{\text {high }}$ T cell population. Eur. J. Immunol. 23:1057-1063.

6. Swain, S. L. 1993. Effector functions of helper T cells. J. Immunother. 14:150-154.

7. Ernst, D. N., W. O. Weigle, D. J. Noonan, D. N. McQuitty, and M. V. Hobbs. 1993. The age-associated increase in IFN-gamma synthesis by mouse $\mathrm{CD}^{+} \mathrm{T}$ cells correlates with shifts in the frequencies of cell subsets defined by membrane CD44, CD45RB, 3G11, and MEL-14 expression. J. Immunol. 151:575-587.

8. Giorgi, J. V., and R. Detels. 1989. T-cell subset alterations in HIV-infected homosexual men: NIAID multicenter AIDS cohort study. Clin. Immunol. Immunopathol. 52:10-18.

9. McGrath, M. S. 1990. T cell Abnormalities. In AIDS Knowledge Base. Textbook on HIV from the University of California, San Francisco and San Francisco General Hospital. P. T. Cohen, M. A. Sande, and P. A. Volberding, editors. Medical Publishing Group, Waltham. 3.2.2-1-3.

10. Rabin, R. L., M. Roederer, Y. Maldonado, A. Petru, L. A. Herzenberg, and L. A. Herzenberg. 1995. Altered representation of naive and memory CD8 T cell subsets in HIV-infected children. J. Clin. Invest. 95:2054-2060.

11. Froebel, K. S., K. V. Doherty, J. A. Whitelaw, R. A. Hague, J. Y. Mok, and A. G. Bird. 1991. Increased expression of the CD45RO (memory) antigen on T cells in HIV-infected children. AIDS (Phila.). 5:97-99.

12. Borkowsky, W., T. Moore, K. Krasinski, K. O. Ajuang-Simbiri, and R. Holzman. 1992. Evolution of phenotypic memory T cells in HIV-1 infected infants and children. Clin. Immunol. Immunopathol. 63:280-284.

13. Reddy, M. M., and M. H. Grieco. 1991. Quantitative changes in T helper inducer $\left(\mathrm{CD}^{+} \mathrm{CD}^{+} \mathrm{RA}^{-}\right)$, $\mathrm{T}$ suppressor inducer $\left(\mathrm{CD4}^{+} \mathrm{CD}^{+} \mathrm{RA}^{+}\right)$, $\mathrm{T}$ suppres-

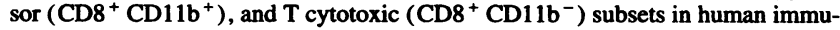
nodeficiency virus infection. J. Clin. Lab. Anal. 5:96-100.

14. Prince, H. E., S. Kleinman, C. Czaplicki, J. John, and A. E. Williams 1990. Interrelationships between serologic markers of immune activation and $T$ lymphocyte subsets in HIV infection. J. Acquired Immune Defic. Syndr. 3:525530.

15. Teitel, J. M., J. J. Freedman, M. B. Garvey, and M. Kardish. 1989. Twoyear evaluation of clinical and laboratory variables of immune function in 117 hemophiliacs seropositive or seronegative for HIV-1. Am. J. Hematol. 32:262272.

16. Moore, W. A., and R. A. Kautz. 1986. Data analysis in flow cytometry. In Handbook of Experimental Immunology. D. M. Weir, L. A. Herzenberg, and L. A. Herzenberg, editors. Blackwell Scientific Publications, Edinburgh. 30.130.11 .

17. Bonyhadi, M. L., L. Rabin, S. Salimi, D. Brown, J. Kosek, J. McCune, and H. Kaneshima. 1993. HIV induces thymus depletion in vivo. Nature (Lond.). 363:728-732.

18. Cook, R. T., T. J. Waldschmidt, Z. K. Ballas, B. L. Cook, B. M. Booth, B. C. Steward, and M. J. Garvey. 1994. Fine T-cell subsets in alcoholics as determined by the expression of L-selectin, leukocyte common antigen, and $\beta$ integrin. Alcohol. Clin. Exp. Res. 18:71-80. 